\title{
Pulmonary Micronodule Detection from 3D Chest CT
}

\author{
Sukmoon Chang ${ }^{1,2}$, Hirosh Emoto ${ }^{3}$, Dimitris N. Metaxas ${ }^{1}$, and Leon Axel ${ }^{4}$ \\ 1 Center for CBIM, Rutgers University, Piscataway, NJ, USA \\ $\{$ sukmoon, dnm\}@cs.rutgers.edu \\ 2 Computer Science, Penn State Capital College, Middletown, PA, USA \\ 3 National Institute of Information and Communications Technology, Tokyo, Japan \\ jiang@nict.go.jp \\ 4 Department of Radiology, New York University, New York, NY, USA \\ leon.axel@med.nyu.edu
}

\begin{abstract}
Computed Tomography (CT) is one of the most sensitive medical imaging modalities for detecting pulmonary nodules. Its high contrast resolution allows the detection of small nodules and thus lung cancer at a very early stage. In this paper, we propose a method for automating nodule detection from high-resolution chest CT images. Our method focuses on the detection of discrete types of granulomatous nodules less than $5 \mathrm{~mm}$ in size using a series of 3D filters. Pulmonary nodules can be anywhere inside the lung, e.g., on lung walls, near vessels, or they may even be penetrated by vessels. For this reason, we first develop a new cylinder filter to suppress vessels and noise. Although nodules usually have higher intensity values than surrounding regions, many malignant nodules are of low contrast. In order not to ignore low contrast nodules, we develop a spherical filter to further enhance nodule intensity values, which is a novel 3D extension of Variable N-Quoit filter. As with most automatic nodule detection methods, our method generates false positive nodules. To address this, we also develop a filter for false positive elimination. Finally, we present promising results of applying our method to various clinical chest CT datasets with over $90 \%$ detection rate.
\end{abstract}

\section{Introduction}

Early detection of lung cancer is critical to improving chances of survival. The five-year survival rate of lung cancer patients is nearly $50 \%$ if lung cancer is found at a localized state (i.e., before it has spread to other organs) and can reach $85 \%$ if it is diagnosed in an early stage and surgery is possible [1,2]. Once the cancer has spread to other organs, the survival rates decline dramatically$20 \%$ at regional stage and $2.2 \%$ at distant stage. Nevertheless, only $15 \%$ of lung cancer cases are found at the localized early stage. For early diagnosis of lung cancer, it is critical to detect nodules less than $5 \mathrm{~mm}$ in size.

Various computational methods have been developed and considerable efforts have been made on automating nodule detection from chest radiographs [16]. 
However, the low sensitivity of chest radiographs to small nodules restricts current systems to the detection of nodules larger than $1 \mathrm{~cm}$ in diameter. Newer medical imaging modalities such as low-dose helical CT allow the detection of pulmonary nodules smaller than those from conventional radiographs. With its high contrast resolution, CT makes it possible to detect nodules of small size or low-contrast that are hard to be seen on conventional radiographs [8].

In this paper, we propose a method for automating nodule detection from high-resolution chest CT images. Our method focuses on the detection of discrete types of granulomatous nodules less than $5 \mathrm{~mm}$ in size using a series of $3 \mathrm{D}$ filters. Since pulmonary nodules can be anywhere inside lung, we first develop a new cylinder filter to suppress vessels and noise. Moreover, noting that many malignant nodules are of low contrast, we develop a spherical filter to further enhance nodule intensity values. Finally, we develop a new filter for false positive elimination. We also present promising results of applying our method to various clinical chest CT datasets.

\section{Previous Work}

Pulmonary nodule detection is one of the most challenging tasks in medical imaging. Various factors can hinder the automatic detection of nodules. Some factors are related to nodule properties, while others are related to the complex lung geometry. Most frequently used properties of nodules in automatic detection are the shape, size, and intensity profile. Template matching techniques were used to explore these features in automated detection of nodules. For example, spherical models with Gaussian distribution in intensity were used as base nodular models for template matching [10. Takizawa et. al. also used cylindrical vascular models along with spherical nodular models in template matching [15. Various pattern recognition techniques have also been used such as fuzzy clustering [7], a linear discriminant classifier 39, rule-based classification [4, and patient-specific a priori model 5 .

A filtering technique called Quoit filter has shown promising results [17. The filter is designed to produce strong response to an isolated circular area. However, it fails when nodules do not match with the filter in size or when nodules are not sufficiently isolated from nearby or penetrating vessels. To remedy the deficiencies of the Quoit filter, Miwa et. al. developed the Variable N-Quoit filter [12. However, their system for nodule detection using the new filter dramatically increased the number of false positives. In addition, their system is overly complicated involving two 2D Quoit filtering and a 3D Quoit filtering processes.

In this paper, we propose a novel and efficient method for automatic detection of granulomatous nodules less than $5 \mathrm{~mm}$ in size from chest CT images. Since nodules can be anywhere inside lung, the automatic nodule detection may be hindered by other structures such as lung walls, nearby vessels, or even penetrating vessels. For this, we first develop a new cylinder filter to suppress vessels and other structures. Although granulomatous nodules frequently have higher 


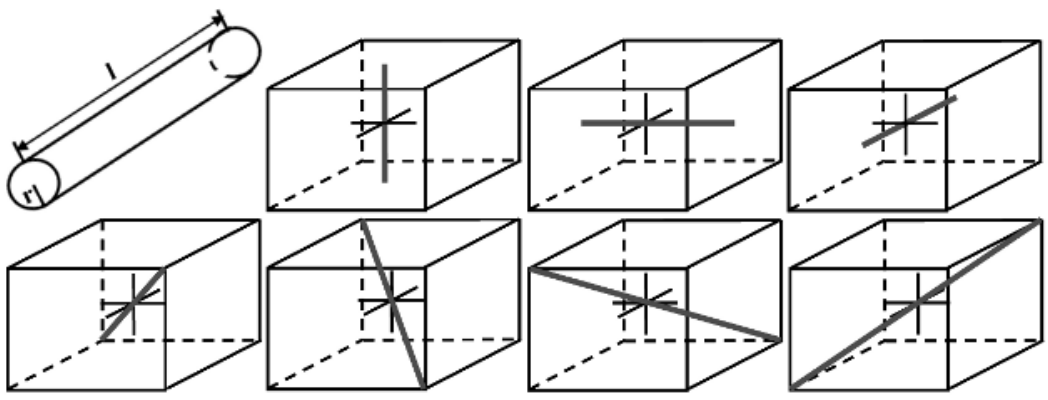

Fig. 1. Bar filter and its orientations.

intensity values than surrounding regions due to extensive calcification, most malignant nodules are noncalcified and, thus, of lower contrast. In order to detect low-contrast noncalcified nodules, we develop a spherical filter to further enhance nodule intensity values. The spherical filter is a novel and straightforward 3D extension of the Variable N-Quoit filter [12. As with most nodule detection methods, our method generates many false positive nodules. Thus, we also develop a new filter for false positive elimination that performs a sphericity test for each candidate nodule. We finally report promising preliminary results of our method applied to clinical chest CT images.

\section{Method}

In this section, we develop a series of 3D filters for automatic micronodule detection from chest CT images. Our primary focus is the detection of discrete types of granulomatous nodules less than $5 \mathrm{~mm}$ in size. Although granulomas usually appear brighter than surrounding regions due to their extensive calcification resulting in a higher X-ray absorption rate, most malignant nodules are noncalcified and, thus, of lower contrast. Our approach to micronodule detection can cope with the aforementioned nodule properties and targets both calcified and noncalcified nodules.

\subsection{Cylinder Filter for Vessel and Noise Suppression}

Nodules can be anywhere inside lung. For example, they can be adjacent to lung walls or fissures, near vessels, or they even can be penetrated by vessels. The performance of any nodule detection method may be hindered by various structures inside lung. To address this difficulty, we first develop a cylinder filter. The cylinder filter is used to suppress intensity values of vessels and other elongated structures inside the lung, while maintaining nodule intensity values intact.

The cylinder filter $F_{\text {cyl }}$ is defined as:

$$
F_{\text {cyl }}(\boldsymbol{x})=\max _{\theta}\left(\min _{\boldsymbol{y} \in \Omega_{\theta}^{\boldsymbol{x}}} I(\boldsymbol{y})\right)
$$




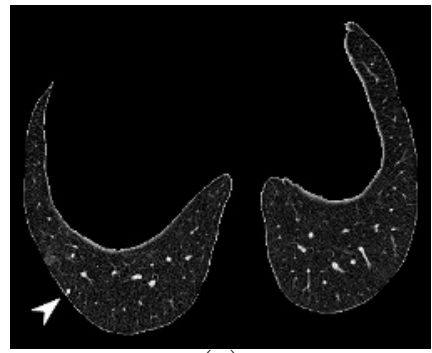

(a)

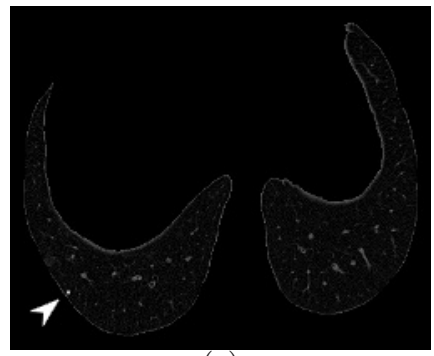

(c)

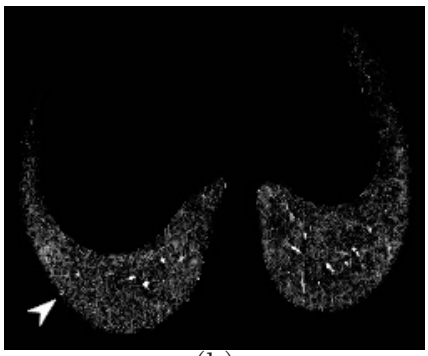

(b)

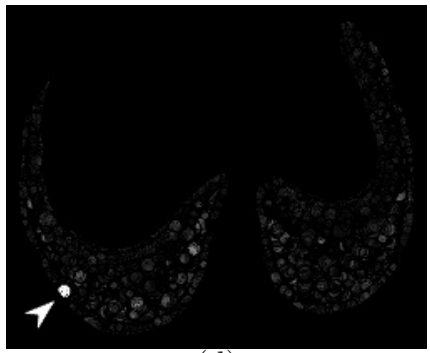

(d)

Fig. 2. Filtered images. (a) Original image $(I)$, (b) Image filtered with $F_{\text {cyl }}$, (c) Image filtered with $I-F_{\text {cyl }}$, and (d) Final image filtered with $F_{\mathrm{sph}}$. The arrow in each image points to the location of a nodule.

where, $\Omega_{\theta}^{\boldsymbol{x}}$ is the domain of the cylinder filter centered at $\boldsymbol{x}$ with orientation $\theta$. $F_{\text {cyl }}$ is a hybrid maxmin neighborhood filter that produces strong responses to cylindrical elongated regions (i.e., vessels). In this paper, we have selected the parameters of $F_{\text {cyl }}$ empirically and used a cylinder with radius of 2 voxels and length of 7 voxels at 7 different orientations, as shown in Fig 1

To suppress vessel intensity values using $F_{\text {cyl }}$, we use

$$
I^{\prime}(\boldsymbol{x})=I(\boldsymbol{x})-F_{\text {cyl }}(\boldsymbol{x})
$$

Applying $F_{\text {cyl }}$ as in (2), the vessel intensity values are effectively suppressed while the nodule intensity values remain almost intact. Fig. 2(b) illustrates the result of applying $F_{\text {cyl }}$ to a dataset containing the original image in (a) Note that $F_{\text {cyl }}$ responded strongly to vessels but weakly to the nodule. By subtracting the two images in (a) and (b) using (2), we obtain a new image shown in (c). In this figure, we can see that vessels and noise are effectively suppressed while the nodule intensity remains intact.

\subsection{Spherical Filter for Nodule Enhancement}

Feature-based approaches for pulmonary nodule detection have shown promising results. The features most widely used are the size, shape, and intensity of nodules 3417. Granular nodules tend to be spherical with higher intensity than surrounding regions. However, many malignant nodules are of relatively 


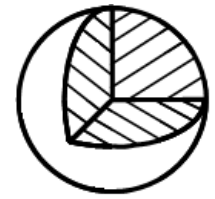

(a)

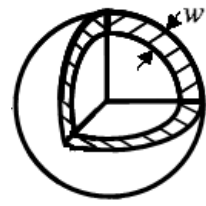

(b)

Fig. 3. Sphere filters. (a) Filled sphere filter $F_{\text {fill }}$ and (b) Hollow sphere filter, $F_{\text {hollow }}$. Domains $\left(\Omega_{\text {fill }}\right.$ and $\Omega_{\text {hollow }}$ ) of the filters are shaded in the figure.

low contrast. In order not to miss low contrast nodules, we develop a spherical filter that enhances the intensity values of nodule areas (i.e., spherical regions with relatively high intensity compared to surrounding regions). To achieve this, we develop a non-linear spherical filter $F_{\mathrm{sph}}$ with two component filters $F_{\text {fill }}$ and $F_{\text {hollow }}$. Let $I(\boldsymbol{x})$ be a $3 \mathrm{D}$ image and $S_{r}(\boldsymbol{x})$ a solid sphere with radius $r$ centered at $\boldsymbol{x}$. Then, the response of the filter $F_{\mathrm{sph}}$ at a point $\boldsymbol{x}$ is

$$
F_{\text {sph }}(\boldsymbol{x})=F_{\text {fill }}(\boldsymbol{x})-F_{\text {hollow }}(\boldsymbol{x})=\max _{\boldsymbol{y} \in \Omega_{\text {fill }}^{\boldsymbol{x}}} I(\boldsymbol{y})-\max _{\boldsymbol{y} \in \Omega_{\text {hollow }}^{\boldsymbol{x}}} I(\boldsymbol{y})
$$

where, $\Omega_{\text {fill }}^{\boldsymbol{x}}$ and $\Omega_{\text {hollow }}^{\boldsymbol{x}}$ are the domains of the filters $F_{\text {fill }}$ and $F_{\text {hollow }}$ centered at $\boldsymbol{x}$, respectively. In other words, as illustrated in Fig. 3 .

$$
\Omega_{\text {fill }}^{\boldsymbol{x}}=S_{r}(\boldsymbol{x}) \quad \text { and } \quad \Omega_{\mathrm{hollow}}^{\boldsymbol{x}}=S_{r}(\boldsymbol{x})-S_{r^{\prime}}(\boldsymbol{x}), \quad r>r^{\prime}
$$

$F_{\mathrm{sph}}$ responds strongly to isolated spherical nodules and weakly to cylindrical vessels. The large differences of the filter responses between nodules and vessel areas allows the automatic detection of pulmonary nodules by a simple thresholding operation. Note that $F_{\mathrm{sph}}$ fails to produce strong responses to nodules when the size of a nodule does not match with the size of $F_{\mathrm{sph}}$. The size of nodules to be detected is determined by the size of $F_{\mathrm{sph}}$. If the filter size is smaller than a nodule, $F_{\text {hollow }}$ is embedded inside the nodule and produces a strong response, weakening the overall response of $F_{\mathrm{sph}}$ from (3). On the other hand, if the filter size is too large, $F_{\text {hollow }}$ may again produce a strong response due to nearby vessels if there are any. To avoid such difficulties, we follow the approach in 12 and employ the adaptive $F_{\mathrm{sph}}$ whose size is optimally adjusted by

$$
r(\boldsymbol{x})=r^{\prime}(\boldsymbol{x})+w \quad \text { and } \quad r^{\prime}(\boldsymbol{x})=\min _{\pi \in \Pi(\boldsymbol{x})}(|\pi|)
$$

where, $\Pi(\boldsymbol{x})$ is the set of all paths from $\boldsymbol{x}$ to the background, $|\pi|$ is the length of a path $\pi$, and $w$ is the width of $F_{\text {hollow }}$ as in Fig. 3. The result of $F_{\mathrm{sph}}$ applied to a cylinder-filtered image is shown in Fig. 2(d). As expected, only the nodule produced strong response to $F_{\mathrm{sph}}$.

\subsection{False Positive Elimination}

The challenging problem for any automatic nodule detection system is to keep the false positive detection rate low while maintaining high sensitivity. Various 
methods have been developed to reduce the false positive detection rates, including feature analysis 311] and template matching 6 10 15. In this paper, we use a sphericity test for each candidate nodules detected by $F_{\mathrm{sph}}$. Note that the detected nodules have already been through the sphericity test in 3D as well as the peak-valley ratio test in the intensity histogram by $F_{\mathrm{sph}}$. Thus, the sphericity test for false positive elimination is performed in a $2 \mathrm{D}$ context.

Let $C$ be a cube surrounding a suspicious nodule area. The intensity values inside $C$ are projected onto $C$ along $x, y$, and $z$-axes by applying MIP [14, generating three $2 \mathrm{D}$ images, $C_{i}, i=1,2,3$. The suspicious nodule area in each of these images is extracted separately by thresholding. In order not to affect the degree of automation, the three threshold values are automatically computed using a threshold selection method such as [13. The sphericity test is then applied to the three segmented nodule areas. Let $A_{i}$ and $L_{i}$ be the area and the border length of $C_{i}$, respectively. Then, the sphericity of the area is tested using

$$
F_{\mathrm{e}}^{i}=\frac{4 \pi A_{i}}{L_{i}^{2}}
$$

Note that $F_{\mathrm{e}}^{i}$ is 1 for a circle and the more elongated the area, the weaker the response of $F_{\mathrm{e}}^{i}$. The suspicious nodules are classified as false positives and eliminated if any of the three segmented nodule areas fails to pass the test.

\section{Experiments}

We applied the method to twelve clinical CT datasets. Each dataset was digitally resliced to ensure cubic voxels and the lung areas were extracted. $F_{\text {cyl }}$ was then applied to each dataset to suppress vessels and noise. We have selected the parameters of $F_{\text {cyl }}$ empirically and used a cylinder with radius of 2 voxels and length of 7 voxels at 7 orientations. The results were filtered again with $F_{\mathrm{sph}}$ to enhance nodule intensities. Then, suspicious nodule regions were extracted by thresholding. Each of the candidate nodules was further processed with $F_{\mathrm{e}}^{i}$ for the sphericity test. Our method reported 69 nodules in all the datasets. An experienced radiologist verified that all the 62 nodules present in the datasets were correctly identified and confirmed that they were less than $5 \mathrm{~mm}$ in diameter. The results are summarized in Table 1 Although our method detected all the 62 nodules present in the datasets, it also reported 7 false positive nodules. These cases were caused by abrupt intensity changes in small regions of vessels, which are very similar to nodules penetrated by vessels.

Fig. 4 shows typical cases of the detected nodules. In each pair of images in this figure, the processed image is shown on the left and the original image on

Table 1. Results.

\begin{tabular}{ccccccc}
\hline Datasets & Nodules(Rad.) & Nodules $\left(F_{\mathrm{sph}}\right)$ & Nodules $\left(F_{\mathrm{e}}^{i}\right)$ & TP & FP & FN \\
\hline 8 & 62 & 127 & 69 & 62 & 7 & 0 \\
\hline
\end{tabular}




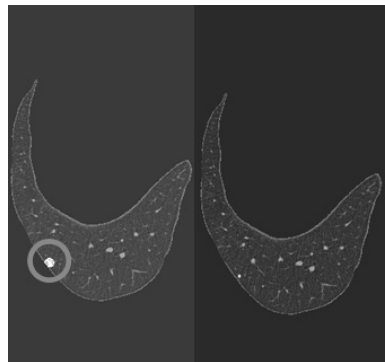

(a)

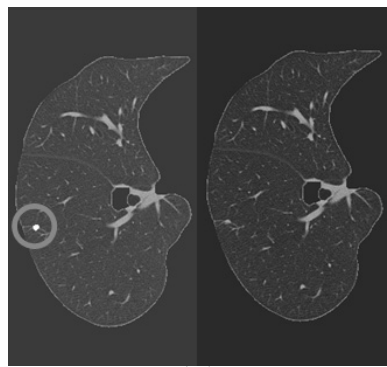

(d)

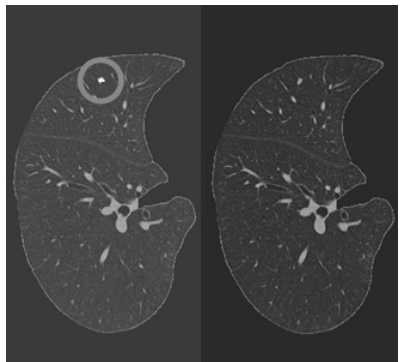

(b)

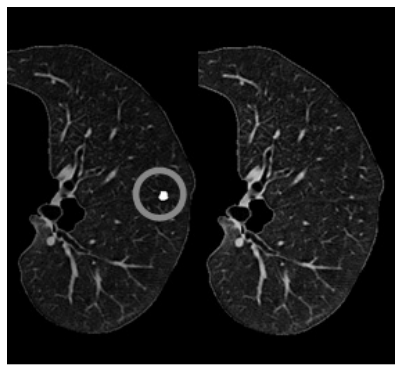

(e)

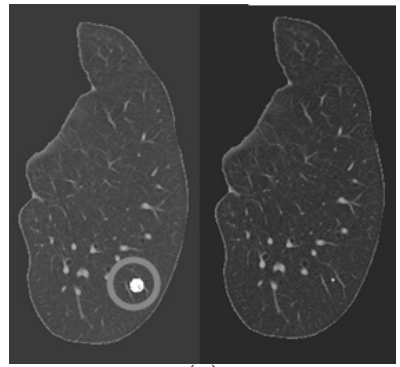

(c)

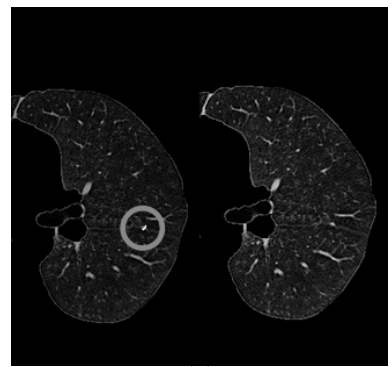

(f)

Fig. 4. Detected nodules. In each image, the processed image is shown on the left and the original image on the right.

the right. The method successfully detected nodules that are close to lung walls (Fig. 4(a)), sufficiently isolated nodules (Fig. 4(b)), nodules with nearby vessels (Fig. 4 (c)), nodules with penetrating vessels (Fig. 4 (d) and (e)), and nodules adjacent to a fissure (Fig. 4 (f)).

\section{Conclusions}

We have introduced a series of filters for automated micronodule detection from 3D chest CT. These include a cylinder filter for vessel suppression, which generates sufficient gaps in intensities between nodule and vessel regions for further processing. Then, the sphere filters were introduced for nodule enhancement, which were natural 3D extensions of $2 \mathrm{D}$ quoit filters. Finally, we proposed a filter for sphericity test for false positive elimination.

We conducted a preliminary set of experiments with the filters on twelve clinical CT datasets. The experiments confirmed that the proposed method was able to detect various nodules including those with nearby vessels or even penetrating vessels and fissures. The datasets contained 62 nodules with size less than $5 \mathrm{~mm}$ and the method detected all of them. However, it also reported 7 false positive cases. These cases result from the abrupt intensity changes on small regions of vessels, which is very hard to differentiate from nodules with penetrating vessels. With the promising preliminary results, we plan to further our experiments in the future to obtain statistically useful validation. 


\section{References}

1. Alliance for Lung Cancer Advocacy, Support, and Education: Early Detection and Diagnostic Imaging (2001)

2. American Cancer Society: Cancer Facts and Figures (2003)

3. Armato, S.G., Giger, M.L., Moran, et. al.: Computerized Detection of Pulmonary Nodules on CT Scans. Radiographics 19 (1999) 1303-1311

4. Betke, M., Ko, J.P.: Detection of Pulmonary Nodules on CT and Volumetric Assessment of Change over Time. MICCAI (1999) 245-252

5. Brown, M.S., McNitt-Gray, M.F., Goldin, et. al.: Patient-Specific Models for Lung Nodule Detection and Surveillance in CT Images. IEEE Trans. Med. Imag. 20:12 (2001) 1242-1250

6. Hara, T., Fujita, H., Goto, S., et. al.: Pattern Recognition Technique for Chest CAD System. Computer-Aided Diagnosis in Medical Imaging (1999) 57-61

7. Kanazawa, K., Kawata, Y., Niki, N., et. al.: Computer-Aided Diagnosis for Pulmonary Nodules based on Helical CT Images. Comput. Med. Imag. Graph. 22:2 (1998) 157-167

8. Kaneko, M., Eguchi, K., et. al.: Peripheral Lung Cancer: Screening and Detection with Low-Dose Spiral CT versus Radiography. Radiology 201 (1996) 798-802

9. Kawata, Y., Niki, N., Ohmatsu, H., et. al: Computer-Aided Diagnosis of Pulmonary Nodules Using Three-Dimensional Thoracic CT Images. MICCAI (2001) 13931394

10. Lee, Y., Hara, T., Fujita, H, Itoh, S., Ishigaki, T.: Automated Detection of Pulmonary Nodules in Helical CT Images Based on an Improved Template-Matching Technique. IEEE Trans. Med. Imag. 20:7 (2001) 595-604

11. McNitt-Gray, M.F., Wyckoff, E., Hart, M., et. al.: Computer Aided Techniques to Characterize Solitary Pulmonary Nodules Imaged on CT. Computer-Aided Diagnosis in Medical Imaging, (1999) 101-106

12. Miwa, T., Kako, J., et. al.: Automatic Detection of Lung Cancers in Chest CT Images by the Variable N-Quoit Filter. Syst. Comput. Jpn. 33:1 (2002) 53-63

13. Otsu, N.: A Threshold Selection Method from Gray-Level Histograms. IEEE Trans. Syst. Man and Cybern. 9:1 (1979) 62-66

14. Sato, Y., Shiraga, N., Nakajima, S., Tamura, S., Kikinis, R.: LMIP: Local Maximum Intensity Projection-A New Rendering Method for Vascular Visualization. J. Comput. Assist. Tomogr. 22:6 (1998) 912-917

15. Takizawa, H., Shigemoto, K., Yamamoto, S.: A Recognition Method of Lung Nodule Shadows in X-Ray CT Images Using 3D Object Models. IJIG 3:4 (2003) 533545

16. van Ginneken, B., ter Haar Romeny, B.M., Viergever, M.A.: Computer-Aided Diagnosis in Chest Radiography: A Survey. IEEE Trans. Med. Imag. 20:12 (2001) 1228-1241

17. Yamamoto, S., Matsumoto, M., Tateno, Y., et. al.: Quoit Filter: A New Filter Based on Mathematical Morphology to Extract the Isolated Shadow, and Its Application to Automatic Detection of Lung Cancer in X-ray CT. ICPR 2 (1996) 3-7 